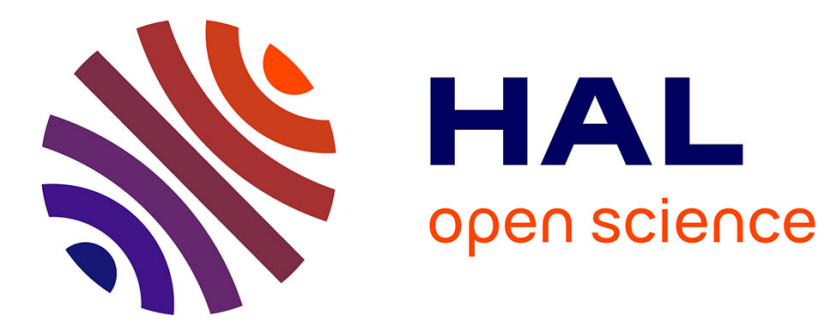

\title{
Traffic models for user-level performance evaluation in data networks
}

Thomas Bonald

\section{To cite this version:}

Thomas Bonald. Traffic models for user-level performance evaluation in data networks. ITC, 2015, Ghent, Belgium. 10.1109/ITC.2015.20 . hal-01243981

\section{HAL Id: hal-01243981 \\ https://hal.inria.fr/hal-01243981}

Submitted on 15 Dec 2015

HAL is a multi-disciplinary open access archive for the deposit and dissemination of scientific research documents, whether they are published or not. The documents may come from teaching and research institutions in France or abroad, or from public or private research centers.
L'archive ouverte pluridisciplinaire HAL, est destinée au dépôt et à la diffusion de documents scientifiques de niveau recherche, publiés ou non, émanant des établissements d'enseignement et de recherche français ou étrangers, des laboratoires publics ou privés. 


\title{
Traffic Models for User-Level Performance Evaluation in Data Networks
}

\author{
Thomas Bonald \\ Telecom ParisTech \\ Paris, France \\ thomas.bonald@telecom-paristech.fr
}

\begin{abstract}
Traffic modeling is key to the capacity planning of data networks. Usual models rely on the implicit assumption that each user generates data flows in series, one after the other, the ongoing flows sharing equitably the considered backhaul link. We relax this assumption and consider the more realistic case where users may generate several data flows in parallel, these flows having to share the user's access line as well. We derive explicit user-level performance metrics like mean throughput and congestion rate in this context, assuming balanced fair sharing between ongoing flows. These results generalize existing ones in that both match in the limit of an infinite number of access lines.
\end{abstract}

Index Terms-Flow-level model, mean throughput, congestion rate, balanced fairness.

\section{INTRODUCTION}

Internet service providers need to predict the impact of traffic load on the quality of service perceived by their customers. This is increasingly important with the advent of high-speed internet access that tends to move congestion from the access to the backhaul, where resources are shared by several users.

Internet traffic is most often modeled at flow level ${ }^{1}$, assuming some ideal bandwidth sharing between ongoing flows [13], [3], [2], [1], [16]. Modeling traffic at packet level proves too complex and is hardly effective, given that users typically perceive quality of service at flow level [10]. In fact, the flow-level models of data networks can be considered as the analogues for the Erlang model of telephone networks and its extensions to multi-rate circuit-switched networks [4]. They have proved essential for both dimensioning [3], [6], [21], [20], [14] and traffic engineering [13], [19], [17].

These models rely on the implicit assumption that each user generates data flows in series, one after the other, so that bandwidth sharing occurs on the considered backhaul link only, and not on the user's access line. In this paper, we relax this assumption and consider the more realistic case where users may generate several data flows in parallel, these flows having to share both the backhaul link and the user's access line. It is not obvious how bandwidth is shared by end-to-end congestion control in this context. We assume bandwidth sharing is balanced fair, which is the only allocation yielding a closed-form stationary distribution of the network state [7]. Balanced fairness in fact provides a very good

Thomas Bonald is a member of LINCS. See www. lincs. fr

${ }^{1} \mathrm{~A}$ flow is here defined as the set of packets having the same 5-tuple: IP source and destination addresses, IP source and destination ports, protocol. approximation of proportional fairness [5], which is considered as representative of the way bandwidth is shared in the Internet [15], [22], [18].

Existing models assume that flows are generated either according to a Poisson process (the so-called infinite-source model) or by $n$ users, each alterning between the active state and the idle state (the so-called finite-source model) [4]; in both cases, each user has at most one flow in progress at any given time. Our model consists of $n$ users, each generating data flows according to a Poisson process; in particular, there is no limit on the number of flows in progress coming from the same user. We refer to this model as the multi-source model, since each user can now be viewed as an independent source of flows.

Since the model allows each user to generate multiple flows in parallel, it is not sufficient to focus on the flow level to evaluate user-level performance. In particular, the throughput of each user is the total throughput of her flows in progress. The corresponding performance results can differ significantly from those obtained under the infinite-source model and the finite-source model. They coincide only in the limit of an infinite number of access lines.

We consider a single backhaul link of fixed capacity (in bit/s) shared by a population of $n$ users. The total throughput of each user is also constrained by the rate of her access line. The models apply equally to the uplink (from the users to the Internet) and to the downlink (from the Internet to the users). All traffic is elastic, meaning that each flow generated by a user corresponds to some data transfer and remains active as long as the corresponding data have not been fully transferred. Due to the insensitivity property, we do not specify the flow size distribution beyond the mean, nor the distribution of the idle times in the finite-source model [4]. We provide formulas that can be used in planning tools or directly by network engineers to get insights into the impact traffic on user-level performance [14].

In the rest of the paper, we first review and extend the results obtained with the infinite-source model and the finitesource model, with a focus on two performance metrics: mean throughput and congestion rate. We then present the multisource model and compare numerically the results derived from the three models in some typical traffic scenarios. 


\section{INFINITE-SOURCE MODEL}

Like the Erlang model for telephone networks, which relies on the assumption of Poisson call arrivals [12], it is common practice to assume Poisson flow arrivals in data networks. This is referred to as the infinite-source model since it corresponds to the finite-source model (presented in the next section) in the limiting case where the number of users $n$ grows to infinity.

\section{A. No access rates}

We start with the simplest case where there is no rate limit at the access: each user has full access to the backhaul link, which is assumed to be equitably shared by ongoing flows. Flows arrive according to a Poisson process of intensity $\lambda$ and have i.i.d. sizes of mean $\sigma$ bits, corresponding to a traffic intensity of $A=\lambda \sigma$ bit/s. Denoting by $C$ the capacity of the backhaul link in bit/s, the link load is $\rho=A / C$. Under the assumption of perfect fair sharing, the traffic model corresponds to an $M / G / 1$ processor-sharing queue of load $\rho$. It is stable if and only if $\rho<1$, in which case the stationary distribution of the number of flows in progress $X$ is given by:

$$
\pi(x)=(1-\rho) \rho^{x} .
$$

It turns out that the stationary distribution seen by a user having a flow in progress is different. Since there are $x$ flows in progress in state $x$, which are assumed to be generated by different users, each active user sees the size-biased probability distribution of the random variable $X$,

$$
\pi^{\prime}(x) \propto x \pi(x) .
$$

Observe that $\pi^{\prime}(0)=0$. We will denote by $P^{\prime}$ and $E^{\prime}$ the corresponding probability measure and expectation, respectively. We derive two key performance metrics on this basis.

Mean throughput: The first performance metric is the mean throughput experienced by users. Assume there are $x$ ongoing flows, with $x>0$. The throughput of each flow is then $C / x$. Thus the mean throughput experienced by users, normalized by the maximum throughput $C$, is given by

$$
\gamma=E^{\prime}\left(\frac{1}{X}\right) .
$$

Replacing $E^{\prime}$ by its expression, we obtain

$$
\gamma=\frac{\sum_{x>0} \pi(x)}{E(X)}=\frac{\rho}{E(X)},
$$

that is

$$
\gamma=1-\rho
$$

Observe that the mean throughput decreases linearly with the link load.

Congestion rate: The second performance metric is the congestion rate, defined as the probability that an active user gets a throughput less than the maximum throughput $C$. Since there is no rate limit at the access, the congestion rate is the probability seen by an active user that there are other active users:

$$
\eta=P^{\prime}(X>1) .
$$

Replacing $E^{\prime}$ by its expression, we obtain

$$
\eta=\frac{\sum_{x>1} x \pi(x)}{E(X)}=1-\frac{P(X=1)}{E(X)},
$$

that is

$$
\eta=\rho(2-\rho) .
$$

As expected, the congestion rate grows from 0 to 1 as the link load grows from 0 to 1 .

\section{B. Same access rates}

We now consider the practically interesting case where each flow has a rate limit $r<C$ corresponding to the capacity of the user's access line in bit/s. For convenience, we assume that the capacity of the backhaul link is some multiple of this access rate, that is $C=m r$ for some integer $m \geq 1$. We denote by $\alpha=A / r$ the traffic intensity expressed in units of the access rate. This would correspond to the mean number of flows if the backhaul link were of infinite capacity. The model corresponds to an $M / G / m$ processor-sharing queue of load $\rho=A / C$. Under the stability condition $\rho<1$, the stationary distribution of the number of flows $X$ is given by:

$$
\pi(x)=\frac{1}{G} \begin{cases}\frac{\alpha^{x}}{x !} & \text { for } x \leq m, \\ \frac{\alpha^{m}}{m !} \rho^{x-m} & \text { for } x>m,\end{cases}
$$

where $G$ denotes the normalization constant:

$$
G=\sum_{x=0}^{m} \frac{\alpha^{x}}{x !}+\frac{\alpha^{m}}{m !} \frac{\rho}{1-\rho} .
$$

Both performance metrics extend to this case. We still denote by $P^{\prime}$ and $E^{\prime}$ the corresponding size-biased probability measure and expectation.

Mean throughput: Assume there are $x$ ongoing flows, with $x>0$. The throughput of each flow is then $\min (r, C / x)$. We deduce the mean throughput seen by users, normalized by the maximum throughput $r$,

$$
\gamma=\mathrm{E}^{\prime}\left(\min \left(1, \frac{m}{X}\right)\right) .
$$

Replacing $E^{\prime}$ by its expression, we obtain

$$
\gamma=\frac{E(\min (X, m))}{E(X)}=\frac{\alpha}{E(X)},
$$

where the second equality follows from work conservation. Finally, we get

$$
\gamma=\frac{m(1-\rho)^{2}}{\pi(m)+m(1-\rho)^{2}} .
$$

Congestion rate: The throughput of a flow is less than its maximum $r$ whenever the number of flows exceeds $m$. We deduce the congestion rate

$$
\eta=P^{\prime}(X>m),
$$

that is

$$
\eta=\frac{\pi(m)(1+m(1-\rho))}{\pi(m)+m(1-\rho)^{2}} .
$$




\section{Different access rates}

Finally, we consider the general case of $K$ different access rates $r_{1}, \ldots, r_{K}$. We denote by $A_{1}, \ldots, A_{K}$ the respective traffic intensities in bit/s generated by each class of users, and by $\alpha_{1}=A_{1} / r_{1}, \ldots, \alpha_{K}=A_{K} / r_{K}$ the traffic intensities expressed in multiples of the access rates; these would correspond to the mean number of flows of each class if the backhaul link were of infinite capacity. The corresponding loads on the backhaul link are $\rho_{1}=A_{1} / C, \ldots, \rho_{K}=A_{K} / C$, and the total load is $\rho=\rho_{1}+\ldots+\rho_{K}$.

Let $X$ be the $K$-dimensional vector of the number of flows of each class in progress. Denote by $\phi_{k}(x)$ the total throughput of class- $k$ users in state $x$. The capacity constraints are

$$
\forall k=1, \ldots, K, \quad \phi_{k}(x) \leq x_{k} r_{k}
$$

and

$$
\sum_{k=1}^{K} \phi_{k}(x) \leq C .
$$

Now let $r$ be the $K$-dimensional vector of access rates. Under balanced fair sharing [9], all users get their maximum throughput, in the sense that $\phi_{k}(x)=x_{k} r_{k}$ for all $k=1, \ldots, K$, if and only if $x . r \leq C$ (the access lines are limiting); otherwise, no user gets her or his maximum throughput and the total throughput is $\sum_{k=1}^{K} \phi_{k}(x)=C$ (the backhaul link is limiting). The stability condition is $\rho<1$ and the vector $X$ has the stationary distribution:

$$
\pi(x)= \begin{cases}\frac{1}{G} \frac{\alpha_{1}^{x_{1}}}{x_{1} !} \cdots \frac{\alpha_{K}^{x_{K}}}{x_{K} !} & \text { for } x . r \leq C, \\ \sum_{k=1}^{K} \rho_{k} \pi\left(x-e_{k}\right) & \text { for } x . r>C,\end{cases}
$$

where $e_{k}$ is the unit vector on component $k$ and $G$ denotes the normalization constant. Here and in the rest of the paper, we adopt the convention that $\pi(x)=0$ for any $x \notin \mathbb{N}^{K}$.

Performance now depends on the user's class. The stationary distribution of $X$ seen by class- $k$ users is

$$
\pi_{k}^{\prime}(x) \propto x_{k} \pi(x) .
$$

We denote by $P_{k}^{\prime}$ and $E_{k}^{\prime}$ the corresponding probability measure and expectation, respectively.

Mean throughput: All class- $k$ users get the same throughput $\phi_{k}(x) / x_{k}$ in any state $x$ such that $x_{k}>0$. We deduce the mean throughput of class- $k$ users, normalized by the maximum throughput $r_{k}$,

$$
\gamma_{k}=E_{k}^{\prime}\left(\frac{\phi_{k}(X)}{X_{k} r_{k}}\right)
$$

Replacing $E_{k}^{\prime}$ by its expression, we obtain

$$
\gamma_{k}=\frac{E\left(\phi_{k}(X)\right)}{E\left(X_{k}\right) r_{k}}=\frac{\alpha_{k}}{E\left(X_{k}\right)},
$$

where the second equality follows from work conservation.
Congestion rate: The throughput of a class- $k$ user is less than its maximum $r_{k}$ in any state $x$ such that $x . r>C$. We deduce:

$$
\eta_{k}=P_{k}^{\prime}(X . r>C)
$$

Both performance metrics can be computed through a recursive formula [9], which is the analogue of the KaufmannRoberts formula for circuit-switched networks.

\section{Numerical results}

Figure 1 shows the mean throughput and congestion rate as a function of the link load $\rho$ when all users have the same access rate $r$ and the backhaul link has capacity $C=m r$ with $m=1,10,100$. For $m=1$, there is actually no access rate limit and users have full access to the backhaul link, as considered in $\S$ II-A.

We observe that performance improves with $m$, as the backhaul link becomes less constraining. For $m=100$, the mean throughput is approximately equal to the access rate until a load of $85 \%$, corresponding to a congestion rate smaller than $5 \%$ (the impact of the backhaul link is negligible), then decreases quickly to 0 , with congestion rate larger than $5 \%$ (the impact of the backhaul link becomes dominant).

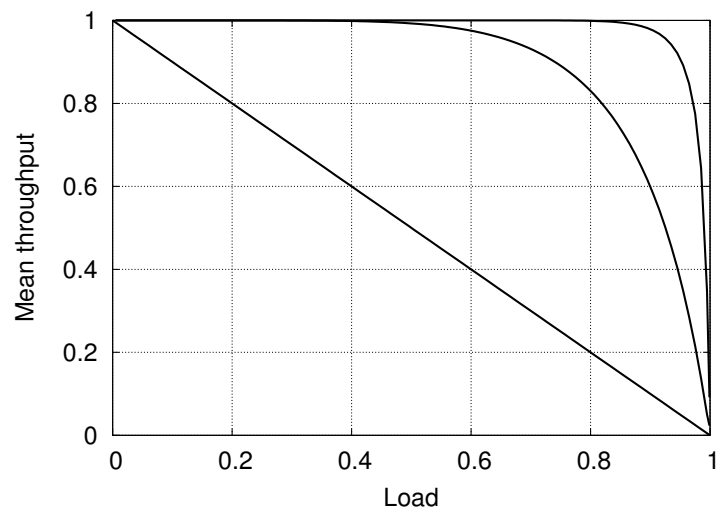

(a) Mean throughput for $m=1,10,100$ (from bottom to top)

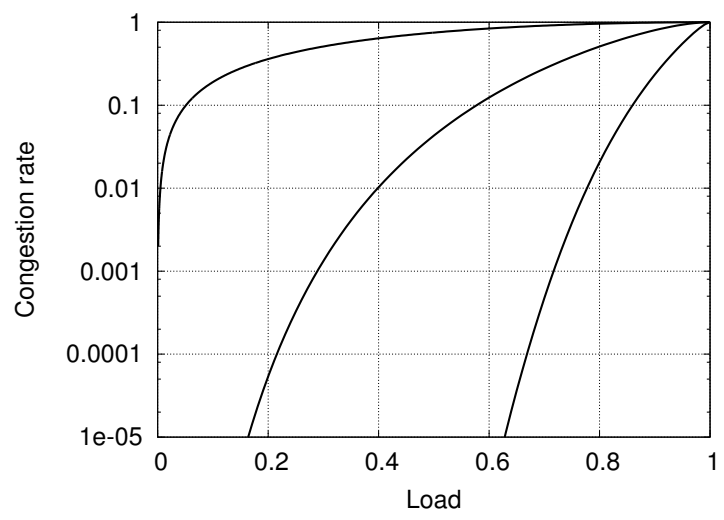

(b) Congestion rate for $m=1,10,100$ (from top to bottom)

Fig. 1. Performance metrics under the infinite-source model. 


\section{FINITE-SOURCE MODEL}

When the user population is relatively small, flow arrivals cannot be considered as Poisson. Each user is still assumed to generate flows in series, with a random idle time between the end of a flow and the beginning of the next flow. This is the analogue of the Engset model used for telephone networks [11]. We only give the stationary distribution of the number of active users; the corresponding performance metrics can be derived as for the infinite-source model.

\section{A. No access rates}

Consider $n$ users having full access to the backhaul link. Any idle user tends to become active at rate $\nu>0$, while any active user tends to become idle at rate $\mu=C / \sigma$ when no other users are active. We deduce that any user alone in the system is active a fraction of time $\beta /(1+\beta)$, with $\beta=\nu / \mu$.

Now assume active users share the backhaul link in a fair way. The stationary distribution of the number of active users $X$ is then given by

$$
\pi(x)=\frac{1}{G} \frac{n !}{(n-x) !} \beta^{x}, \quad x \leq n,
$$

where $G$ is the normalization constant:

$$
G=\sum_{x=0}^{n} \frac{n !}{(n-x) !} \beta^{x} .
$$

The infinite-source model corresponds to the case $n \rightarrow \infty$ and $\beta \rightarrow 0$, with $n \beta \rightarrow \rho$. A key difference with the infinitesource model is that traffic intensity is no longer an exogenous parameter but given by $A=C P(X>0)$. We deduce the link load:

$$
\rho=P(X>0)=\frac{G-1}{G} .
$$

\section{B. Same access rates}

Now assume all users have the same access rate $r$. The link capacity is $C=m r$ for some integer $m \geq 1$, with $n>m$. Any active user tends to become idle at rate $\mu=r / \sigma$ when no other users are active. The stationary distribution of the number of flows $X$ becomes:

$$
\pi(x)=\frac{1}{G} \begin{cases}\left(\begin{array}{l}
n \\
x
\end{array}\right) \beta^{x} & \text { for } x \leq m, \\
\frac{n !}{(n-x) ! m !} \frac{\beta^{x}}{m^{x-m}} & \text { for } m<x \leq n,\end{cases}
$$

where $\beta=\nu / \mu$ and $G$ is the normalization constant. Traffic intensity is $A=E(\min (X, m)) r$, corresponding to load

$$
\rho=\frac{E(\min (X, m))}{m} .
$$

\section{Different access rates}

Finally, consider the general case of $K$ different access rates $r_{1}, \ldots, r_{K}$. There are $n_{k}$ users with access rate $r_{k}$, mean flow size $\sigma_{k}$ and mean idle time $1 / \nu_{k}$ between two flows. Under balanced fair sharing, the stationary distribution of the system state $X$ is given by:

$$
\begin{aligned}
& \pi(x)= \\
& \begin{cases}\frac{1}{G} \prod_{k=1}^{K}\left(\begin{array}{l}
n_{k} \\
x_{k}
\end{array}\right) \beta_{k}^{x_{k}} & \text { for } x . r \leq C, \\
\sum_{k=1}^{K} \frac{\beta_{k} r_{k}}{C}\left(n_{k}-x_{k}+1\right) \pi\left(x-e_{k}\right) & \text { for } x . r>C,\end{cases}
\end{aligned}
$$

where $\beta_{k}=\nu_{k} / \mu_{k}, \mu_{k}=r_{k} / \sigma_{k}$ and $G$ the normalization constant. Traffic intensity is $A=E(\min (X . r, C))$, corresponding to load:

$$
\rho=\frac{E(\min (X \cdot r, C))}{C} .
$$

\section{Numerical results}

Figure 2 shows the mean throughput and the congestion rate when all users have the same access rate $r$ and the backhaul link has capacity $C=m r$ with $m=10$; the number of users is $n=20,50,100$ and $\infty$, this last case corresponding to the infinite-source model. We observe that, while the congestion rate obtained with the finite-source model quickly tends to that derived from the infinite-source model when $n$ grows, the convergence is much slower for the mean throughput: for $n=100$, the results still differ significantly.

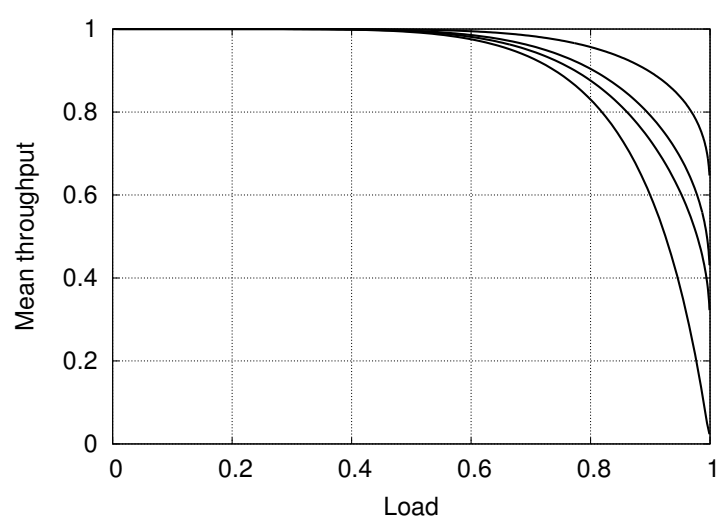

(a) Mean throughput for $m=10$ and $n=20,50,100, \infty$ (from top to bottom)

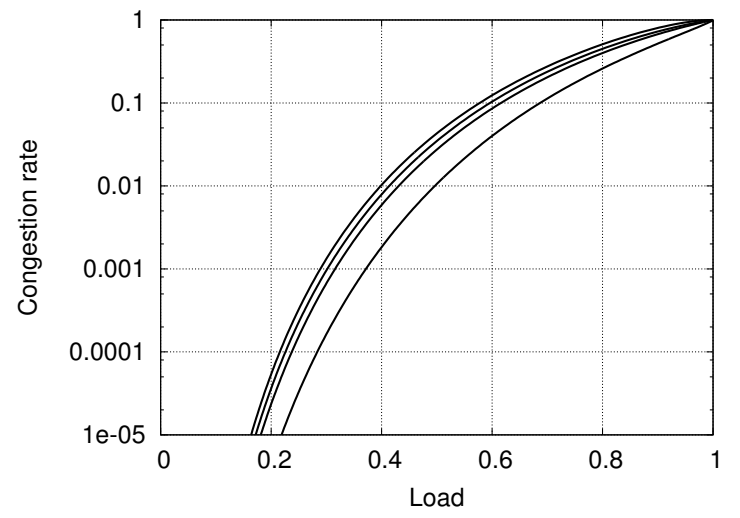

(b) Congestion rate for $m=10$ and $n=20,50,100, \infty$ (from bottom to top)

Fig. 2. Performance metrics under the finite-source model. 


\section{MULTI-SOURCE MODEL}

We now introduce the multi-source model where data flows must share both the backhaul link and the user's access line. We consider $n$ users, with user $i$ generating flows according to an independent Poisson process of intensity $\lambda_{i}$, corresponding to the traffic intensity $a_{i}=\lambda_{i} \sigma$ in bit/s. We are interested in the total throughput obtained by each user.

\section{A. No access rates}

As above, we first consider the case where each user has full access to the backhaul link. Under fair sharing between flows in progress, the model reduces to an $M / G / 1$ multi-class processor-sharing queue. Denoting by $\rho_{i}=a_{i} / C$ the load due to user $i$ and by $\rho=\rho_{1}+\ldots+\rho_{n}$ the total load, the stationary distribution of the number of flows of each user $X$ is given by

$$
\pi(x)=(1-\rho)\left(\begin{array}{c}
x_{1}+\ldots+x_{n} \\
x_{1}, \ldots, x_{n}
\end{array}\right) \rho_{1}^{x_{1}} \ldots \rho_{n}^{x_{n}},
$$

under the stability condition $\rho<1$.

Now user $i$ sees the stationary distribution

$$
\pi_{i}(x) \propto \pi(x) 1_{x_{i}>0}
$$

when active. We denote by $P_{i}$ and $E_{i}$ the corresponding probability measure and expectation.

Mean throughput: The total throughput of user $i$ is proportional to the number of ongoing flows of this user, that is $\left(x_{i} / \sum_{j} x_{j}\right) \times C$ in any state $x$ such that $x_{i}>0$. We deduce the mean throughput of user $i$, normalized by the maximum throughput $C$,

$$
\gamma_{i}=E_{i}\left(\frac{X_{i}}{\sum_{j} X_{j}}\right) .
$$

By work conservation,

$$
\rho_{i}=E\left(\frac{X_{i}}{\sum_{j} X_{j}} 1_{X_{i}>0}\right),
$$

so that

$$
\gamma_{i}=\frac{\rho_{i}}{P\left(X_{i}>0\right)} .
$$

Since

$$
P\left(X_{i}>0\right)=\frac{\rho_{i}}{1-\rho+\rho_{i}},
$$

we obtain

$$
\gamma_{i}=1-\rho+\rho_{i} .
$$

Note that the mean throughput is larger than that obtained with the infinite-source model, given by (1), with equality when $\rho_{i} \rightarrow 0$ (in which case user $i$ generates flows in series, as in the infinite-source model). Observe also that the mean (normalized) throughput of user $i$ is larger than the load $\rho_{i}$ generated by this user, with equality when $\rho \rightarrow 1$ (in which case the system is saturated and the throughput of each user corresponds to her bandwidth share). For homogeneous traffic distribution, all users get the same throughput,

$$
\gamma=1-\rho+\frac{\rho}{n} \geq \frac{1}{n} .
$$

Congestion rate: The congestion rate seen by user $i$ is the probability that the total throughput of this user is less than $C$, that is the probability that there are other active users:

$$
\eta_{i}=P_{i}\left(\sum_{j} X_{j}>X_{i}\right) .
$$

We get

$$
\eta_{i}=\frac{P\left(X_{i}>0, \sum_{j \neq i} X_{j}>0\right)}{P\left(X_{i}>0\right)} .
$$

Since

$$
P\left(X_{i}>0, \sum_{j \neq i} X_{j}=0\right)=\frac{\rho_{i}(1-\rho)}{1-\rho_{i}},
$$

we obtain

$$
\eta_{i}=(2-\rho) \frac{\rho-\rho_{i}}{1-\rho_{i}} .
$$

This congestion rate is smaller than that obtained with the infinite-source model, given by (2), with equality when $\rho_{i} \rightarrow$ 0.

\section{B. Same access rates}

Now assume all users have the same access rate $r$, with $C=m r$ for some integer $m$ such that $1 \leq m<n$. We denote by $\varrho_{i}=a_{i} / r$ the load of user- $i$ access line. The load of user $i$ on the backhaul link is $\rho_{i}=a_{i} / C=\varrho_{i} / \mathrm{m}$.

Let $\phi_{i}(x)$ be the total throughput of user $i$ in state $x$. The capacity constraints are

$$
\forall i=1, \ldots, n, \quad \phi_{i}(x) \leq r
$$

and

$$
\sum_{i=1}^{n} \phi_{i}(x) \leq C .
$$

Let $n(x)=\sum_{i=1}^{n} 1_{x_{i}>0}$ be the number of active users in state $x$. Under balanced fair sharing, all active users get their maximum throughput, that is $\phi_{i}(x)=r$ for all $i=1, \ldots, n$ such that $x_{i}>0$, if and only if $n(x) r \leq C$ (the access lines are limiting); otherwise, no user gets the maximum throughput and the total throughput is $\sum_{i=1}^{n} \phi_{i}(x)=C$ (the backhaul link is limiting). Under the stability condition $\rho<1$ and $\varrho_{i}<1$ for all $i=1, \ldots, n$, the stationary distribution of the network state $X$ is

$$
\pi(x)= \begin{cases}\frac{1}{G} \prod_{i=1}^{n} \varrho_{i}^{x_{i}} & \text { for } n(x) \leq m \\ \sum_{i=1}^{n} \rho_{i} \pi\left(x-e_{i}\right) & \text { otherwise }\end{cases}
$$

where $G$ is the normalization constant. Since the network has a tree topology, we deduce from [8] that

$$
\begin{aligned}
G & =\sum_{I \subset\{1, \ldots, n\},|I| \leq m} \prod_{i \in I} \frac{\varrho_{i}}{1-\varrho_{i}} \\
& +\sum_{I \subset\{1, \ldots, n\},|I|=m} \prod_{i \in I} \frac{\varrho_{i}}{1-\varrho_{i}} \frac{\sum_{i \notin I} \varrho_{i}}{m-\sum_{i=1}^{n} \varrho_{i}} .
\end{aligned}
$$


Mean throughput: The mean throughput of user $i$, normalized by the maximum throughput $r$, is given by

$$
\gamma_{i}=E_{i}\left(\frac{\phi_{i}(X)}{r}\right) \text {. }
$$

By work conservation, $E\left(\phi_{i}(X)\right)=a_{i}$ so that

$$
\gamma_{i}=\frac{\varrho_{i}}{P\left(X_{i}>0\right)}
$$

Now

$$
P\left(X_{i}=0\right)=\frac{G_{i}}{G},
$$

where $G_{i}$ denote the normalization constant in the absence of user $i$,

$$
\begin{aligned}
G_{i} & =\sum_{I \subset\{1, \ldots, n\} \backslash\{i\},|I| \leq m} \prod_{j \in I} \frac{\varrho_{j}}{1-\varrho_{j}} \\
& +\sum_{I \subset\{1, \ldots, n\} \backslash\{i\},|I|=m} \prod_{j \in I} \frac{\varrho_{j}}{1-\varrho_{j}} \frac{\sum_{j \notin I, j \neq i} \varrho_{j}}{m-\sum_{j \neq i} \varrho_{j}} .
\end{aligned}
$$

We deduce

$$
\gamma_{i}=\frac{G \varrho_{i}}{G-G_{i}}
$$

Congestion rate: The congestion rate seen by user $i$ is

$$
\eta_{i}=P_{i}\left(\phi_{i}(X)<r\right) \text {. }
$$

We get

$$
\eta_{i}=\frac{P\left(X_{i}>0, \sum_{j} 1_{X_{j}>0}>m\right)}{P\left(X_{i}>0\right)},
$$

that is

$$
\eta_{i}=\frac{F-F_{i}}{G-G_{i}}
$$

with

$$
F=\sum_{I \subset\{1, \ldots, n\},|I|=m} \prod_{j \in I} \frac{\varrho_{j}}{1-\varrho_{j}} \frac{\sum_{j \notin I} \varrho_{j}}{m-\sum_{j=1}^{n} \varrho_{j}}
$$

and

$$
F_{i}=\sum_{I \subset\{1, \ldots, n\} \backslash\{i\},|I|=m} \prod_{j \in I} \frac{\varrho_{j}}{1-\varrho_{j}} \frac{\sum_{j \notin I, j \neq i} \varrho_{j}}{m-\sum_{j \neq i} \varrho_{j}} .
$$

\section{Different access rates}

We now consider the general case where user $i$ has access rate $r_{i}$. The load of user- $i$ access line becomes $\varrho_{i}=a_{i} / r_{i}$. The capacity constraints are

$$
\forall i=1, \ldots, n, \quad \phi_{i}(x) \leq r_{i}
$$

and

$$
\sum_{i=1}^{n} \phi_{i}(x) \leq C
$$

Under balanced fair sharing, all active users get their maximum throughput if and only if $\sum_{i=1}^{n} r_{i} 1_{x_{i}>0} \leq C$. Under the stability condition $\rho<1$ and $\varrho_{i}<1$ for all $i=1, \ldots, n$, the stationary distribution of the network state $X$ is

$$
\pi(x)= \begin{cases}\frac{1}{G} \prod_{i=1}^{n} \varrho_{i}^{x_{i}} & \text { for } \sum_{i=1}^{n} r_{i} 1_{x_{i}>0} \leq C \\ \sum_{i=1}^{n} \rho_{i} \pi\left(x-e_{i}\right) & \text { otherwise }\end{cases}
$$

where $G$ denotes the normalization constant [8]

$$
\begin{aligned}
G & =\sum_{I \subset\{1, \ldots, n\}, e_{I} . r \leq C} \prod_{i \in I} \frac{\varrho_{i}}{1-\varrho_{i}} \\
& +\sum_{I \subset\{1, \ldots, n\}, e_{I} . r \leq C} \prod_{i \in I} \frac{\varrho_{i}}{1-\varrho_{i}} \frac{\sum_{i \notin I, r_{i}+e_{I} . r>C} \varrho_{i} r_{i}}{C-\sum_{i=1}^{n} \varrho_{i} r_{i}},
\end{aligned}
$$

with $e_{I}$ the vector of ones on all components $i \in I$ and zeros elsewhere.

Mean throughput: The mean throughput of user $i$ normalized by the maximum throughput $r_{i}$ of this user is

$$
\gamma_{i}=E_{i}\left(\frac{\phi_{i}(X)}{r_{i}}\right)
$$

We obtain the same expression (5), with

$$
\begin{aligned}
G_{i} & =\sum_{I \subset\{1, \ldots, n\}, i \notin I, e_{I} . r \leq C} \prod_{j \in I} \frac{\varrho_{j}}{1-\varrho_{j}} \\
+ & \sum_{I \subset\{1, \ldots, n\}, i \notin I, e_{I} . r \leq C} \prod_{j \in I} \frac{\varrho_{j}}{1-\varrho_{j}} \frac{\sum_{j \notin I, j \neq i, r_{j}+e_{I} . r>C} \varrho_{j} r_{j}}{C-\sum_{j \neq i} \varrho_{j} r_{j}} .
\end{aligned}
$$

Congestion rate: The congestion rate seen by user $i$ is

$$
\eta=P_{i}\left(\phi_{i}(X)<r_{i}\right)
$$

that is (6) with

$$
F=\sum_{I \subset\{1, \ldots, n\}, e_{I} . r \leq C} \prod_{j \in I} \frac{\varrho_{j}}{1-\varrho_{j}} \frac{\sum_{j \notin I, r_{j}+e_{I} . r>C} \varrho_{j} r_{j}}{C-\sum_{j} \varrho_{j} r_{j}}
$$

and

$F_{i}=$

$$
\sum_{I \subset\{1, \ldots, n\}, i \notin I, e_{I} . r \leq C} \prod_{j \in I} \frac{\varrho_{j}}{1-\varrho_{j}} \frac{\sum_{j \notin I, j \neq i, r_{j}+e_{I} . r>C} \varrho_{j} r_{j}}{C-\sum_{j \neq i} \varrho_{j} r_{j}} .
$$

\section{Different user classes}

The previous formulas have exponential complexity in $n$. To keep the complexity polynomial in $n$, we need to group users in some finite number of classes $K$, as in the infinite-source model and the finite-source model. With some slight abuse of notation, we denote respectively by $r_{k}$ and $\varrho_{k}$ the rate and the load of the access line of each class- $k$ user. There are $n_{k}$ class- $k$ users and we denote by $n$ the vector $\left(n_{1}, \ldots, n_{K}\right)$. The normalization constant is then given by

$$
\begin{aligned}
G & =\sum_{\ell \leq n: \ell . r \leq C} \prod_{k=1}^{K}\left(\begin{array}{c}
n_{k} \\
\ell_{k}
\end{array}\right)\left(\frac{\varrho_{k}}{1-\varrho_{k}}\right)^{\ell_{k}} \\
& +\sum_{\ell \leq n: \ell . r \leq C} \prod_{k=1}^{K}\left(\begin{array}{c}
n_{k} \\
\ell_{k}
\end{array}\right)\left(\frac{\varrho_{k}}{1-\varrho_{k}}\right)^{\ell_{k}} \\
& \times \frac{\sum_{j: \ell_{j}<n_{j}, \ell . r+r_{j}>C}\left(n_{j}-\ell_{j}\right) \varrho_{j} r_{j}}{C-\sum_{k=1}^{K} n_{k} \varrho_{k} r_{k}} .
\end{aligned}
$$

The mean throughput of each class- $k$ user is

$$
\gamma_{k}=\frac{G \varrho_{k}}{G-G_{k}}
$$


where $G_{k}$ is the constant $\mathrm{G}$ with $n_{k}$ replaced by $n_{k}-1$. Similarly, letting

$$
\begin{aligned}
F & =\sum_{\ell \leq n: \ell . r \leq C} \prod_{k=1}^{K}\left(\begin{array}{c}
n_{k} \\
\ell_{k}
\end{array}\right)\left(\frac{\varrho_{k}}{1-\varrho_{k}}\right)^{\ell_{k}} \\
& \times \frac{\sum_{j: \ell_{j}<n_{j}, \ell . r+r_{j}>C}\left(n_{j}-\ell_{j}\right) \varrho_{j} r_{j}}{C-\sum_{k=1}^{K} n_{k} \varrho_{k} r_{k}},
\end{aligned}
$$

we obtain the congestion rate of class- $k$ users

$$
\eta_{k}=\frac{F-F_{k}}{G-G_{k}}
$$

where $F_{k}$ is the constant $F$ with $n_{k}$ replaced by $n_{k}-1$.

In the limit where the number of users tends to infinity with traffic intensities $n_{1} \varrho_{1} r_{1}, \ldots, n_{K} \varrho_{K} r_{K}$ tending to some fixed constants $A_{1}, \ldots, A_{K}$ such that $A_{1}+\ldots+A_{K}<C$, the multi-source model reduces to the infinite-source model: there is an infinite population of users, each user generating flows according to a Poisson process of null intensity.

\section{E. Numerical results}

Figures 3 and 4 compare the performance metrics obtained with the three considered models when all users have the same access rate $r, C=m r$ with $m=1,10,100, n=2 m$ and $n=10 m$, respectively. Traffic distribution is homogeneous.

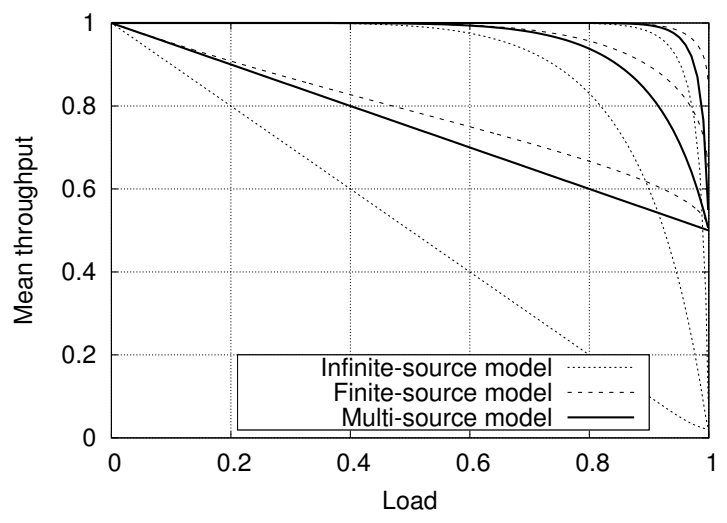

(a) Mean throughput for $m=1,10,100$ (from bottom to top)

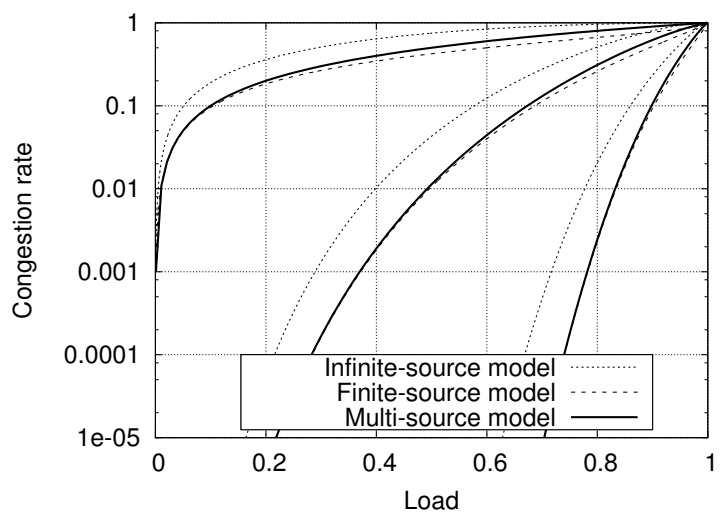

(b) Congestion rate for $m=1,10,100$ (from top to bottom)

Fig. 3. Performance metrics under the three models for $n=2 m$.

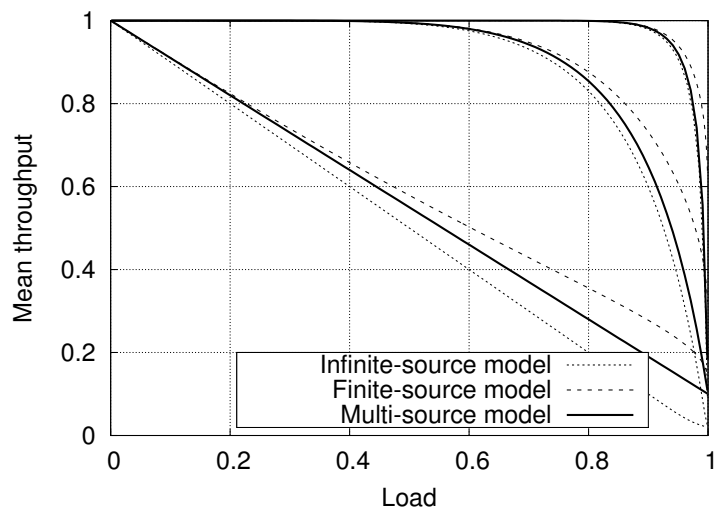

(a) Mean throughput for $m=1,10,100$ (from bottom to top)

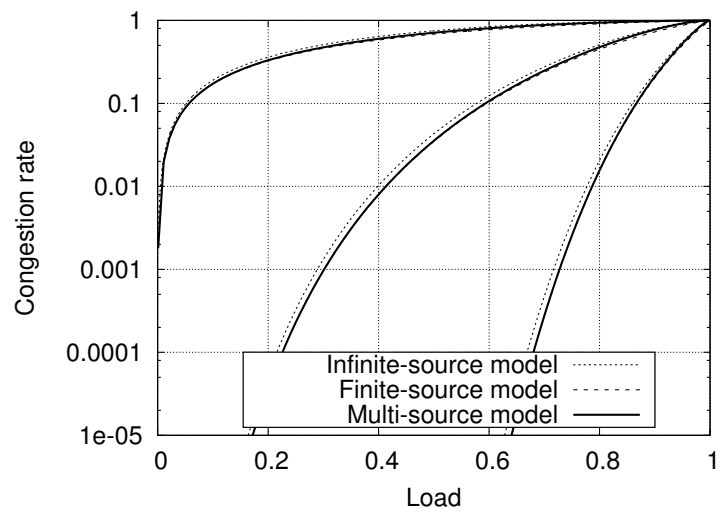

(b) Congestion rate for $m=1,10,100$ (from top to bottom)

Fig. 4. Performance metrics under the three models for $n=10 \mathrm{~m}$.

We observe that the infinite-source model is overly pessimistic while the finite-source model is overly optimistic compared to the multi-source model, especially for the mean throughput. For the congestion rate, the infinite-source model is a good approximation of the multi-source model only for $n=10 \mathrm{~m}$ while the finite-source model is a very good approximation in both cases.

Figure 5 shows the results obtained for two classes of users, class-2 users generating 10 times more traffic than class1 users. There is the same number of class- 1 and class- 2 users. All users have the same access rate $r, C=m r$ with $m=1,10,100$, and there is a total of $n=10 m$ users. We observe that class-1 performance is well approximated by the infinite-source model while class-2 performance is better approximated by the finite-source model. None of these two models is satisfactory: the underlying assumption of flows generated in series by each user does not allow a proper evaluation of user-level performance.

The same conclusion can be drawn from Figure 6, showing the results for two classes of users with different access rates, $r_{1}=1$ and $r_{2}=4$. We take $n_{1}=4 n_{2}$ and the same load for all access lines so that the total traffic intensity is the same for each class. Here $m=C / r_{1}$ takes the values $5,50,500$ and the total number of users is $n=2 m$. 


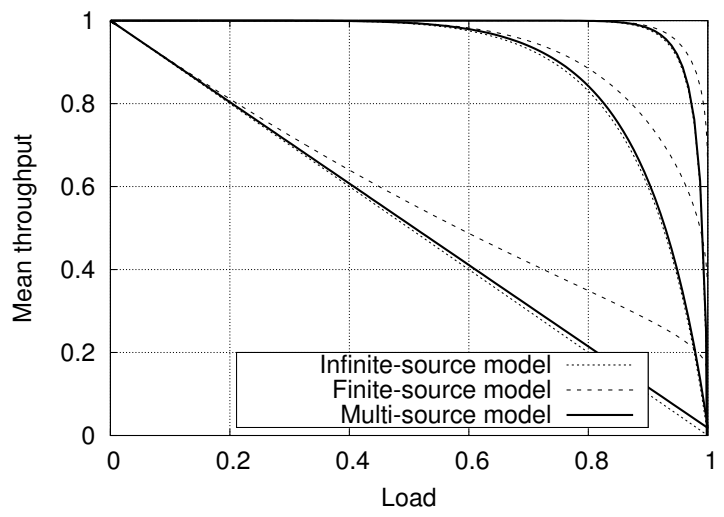

(a) Class1 (light traffic)

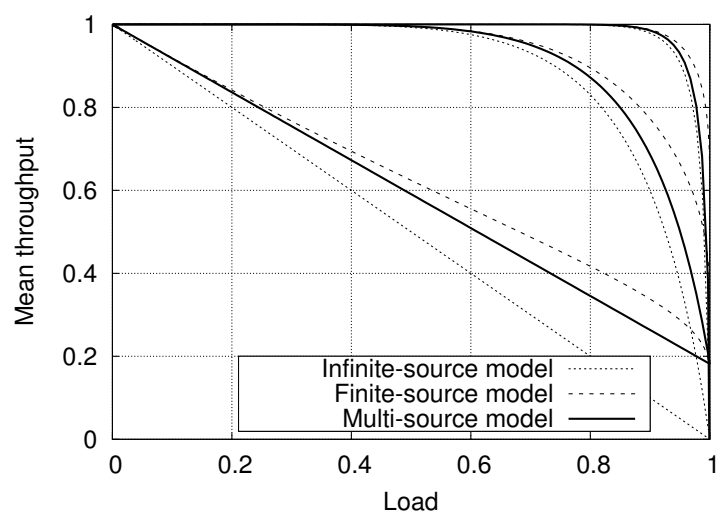

(b) Class 2 (heavy traffic)

Fig. 5. Mean throughput for $m=1,10,100$ (from bottom to top) and $n_{1}=n_{2}=5 m$.

\section{CONCLUSiON}

We have proposed a new traffic model for evaluating userlevel performance in data networks. The key characteristic of this model is to account for bandwidth sharing on the user's access line. The results turn out to be very different from those obtained with usual models in practically interesting cases, like $n=100$ users having different traffic profiles or access rates. They coincide only for large values of $n$, say $n \geq 1000$.

One of the key benefits of the proposed multi-source model is to account precisely for the number of access lines $n$ without the complexity of the finite-source model. For instance, traffic intensity (and thus link load) is an exogenous parameter of the multi-source model but an endogenous parameter of the finite-source model. Moreover, the normalization constant is explicit in the multi-source model, which greatly simplifies the computation of the performance metrics.

A drawback of the multi-source model compared to the infinite-source model is the lack of a recursive formula for evaluating the normalization constant in the presence of a large number of different access rates. We let this for future work. Other interesting issues include the extensions of this model to non-elastic traffic (for instance, adaptive streaming traffic) and to several aggregation stages in the backhaul network.

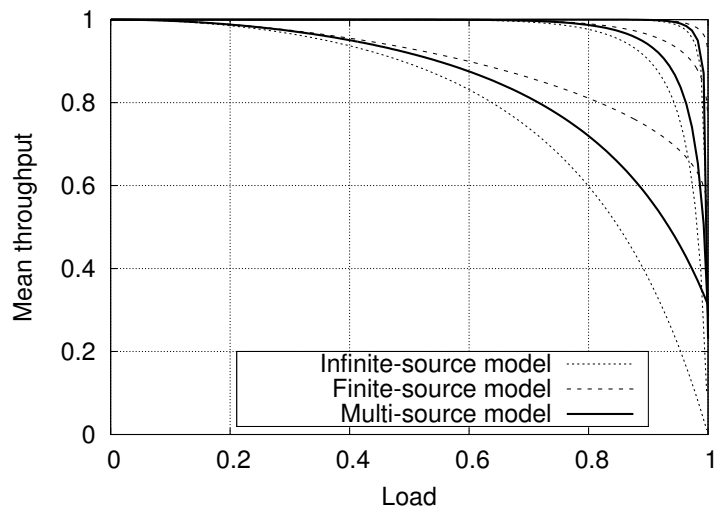

(a) Class 1 (low access rate, $r_{1}=1$ )

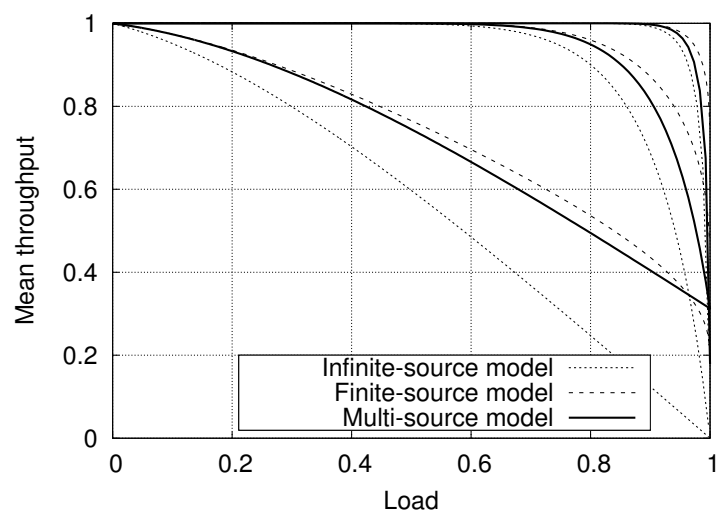

(b) Class 2 (high access rate, $r_{2}=4$ )

Fig. 6. Mean normalized throughput for $m=5,50,500$ (from bottom to top), $n=2 m, n_{1}=4 n_{2}$.

\section{REFERENCES}

[1] C. Barakat, P. Thiran, G. Iannaccone, C. Diot, and P. Owezarski Modeling internet backbone traffic at the flow level. Signal Processing, IEEE Transactions on, 51(8):2111-2124, 2003.

[2] S. Ben Fredj, T. Bonald, A. Proutière, G. Régnié, and J. W. Roberts. Statistical bandwidth sharing: a study of congestion at flow level. In ACM SIGCOMM Computer Communication Review, volume 31, pages 111-122. ACM, 2001.

[3] A. W. Berger and Y. Kogan. Dimensioning bandwidth for elastic traffic in high-speed data networks. IEEE/ACM Trans. Netw., 8(5):643-654, Oct. 2000.

[4] T. Bonald and M. Feuillet. Network Performance Analysis. Wiley, 2011.

[5] T. Bonald, L. Massoulié, A. Proutière, and J. Virtamo. A queueing analysis of max-min fairness, proportional fairness and balanced fairness. Queueing systems, 53(1-2):65-84, 2006.

[6] T. Bonald, P. Olivier, and J. Roberts. Dimensioning high speed IP access networks. In proceedings of the 8th International Teletraffic Congress (ITC), pages 241-251, 2003.

[7] T. Bonald and A. Proutière. Insensitive bandwidth sharing in data networks. Queueing systems, 44(1):69-100, 2003.

[8] T. Bonald, A. Proutière, J. Roberts, and J. Virtamo. Computational aspects of balanced fairness. Teletraffic Science and Engineering, 5:801$810,2003$.

[9] T. Bonald and J. Virtamo. A recursive formula for multirate systems with elastic traffic. IEEE Communications Letters, 9(8):753-755, 2005.

[10] N. Dukkipati and N. McKeown. Why flow-completion time is the right metric for congestion control. ACM SIGCOMM Computer Communication Review, 36(1):59-62, 2006.

[11] T. Engset. On the calculation of switches in an automatic telephone system. In A. Myskja and O. Espvik, editors, Tore Olaus Engset: The man behind the formula, 1998. 
[12] A. Erlang. Solution of some problems in the theory of probabilities of significance in automatic telephone exchanges. In E. Brockmeyer, H. Halstrom, and A. Jensen, editors, The life and works of A.K. Erlang, 1948.

[13] D. P. Heyman, T. Lakshman, and A. L. Neidhardt. A new method for analysing feedback-based protocols with applications to engineering web traffic over the internet. In ACM Sigmetrics performance evaluation review, volume 25, pages 24-38. ACM, 1997.

[14] R. Indre, P. Olivier, B. Kauffmann, and N. Benameur. Evaluating user-perceived performance in high-speed backhaul networks. In 16th International Telecommunications Network Strategy and Planning Symposium (Networks 2014), 2014.

[15] F. P. Kelly, A. K. Maulloo, and D. K. Tan. Rate control for communication networks: shadow prices, proportional fairness and stability. Journal of the Operational Research society, pages 237-252, 1998.

[16] A. A. Kherani and A. Kumar. Stochastic models for throughput analysis of randomly arriving elastic flows in the internet. In INFOCOM 2002, volume 2, pages 1014-1023. IEEE, 2002.
[17] A. Kortebi, L. Muscariello, S. Oueslati, and J. Roberts. On the scalability of fair queueing. In ACM HotNets-III, 2004.

[18] S. Kunniyur and R. Srikant. End-to-end congestion control schemes: Utility functions, random losses and ecn marks. IEEE/ACM Transactions on Networking, 11(5):689-702, 2003.

[19] A. Lakshmikantha, C. Beck, and R. Srikant. Impact of file arrivals and departures on buffer sizing in core routers. IEEE/ACM Transactions on Networking (TON), 19(2):347-358, 2011.

[20] P. Lassila, A. Penttinen, and J. Virtamo. Dimensioning methods for data networks with flow-level QoS requirements. In Local Computer Networks, 2007. LCN 2007. 32nd IEEE Conference on, pages 353-359, Oct 2007.

[21] P. Lassila, A. Penttinen, and J. Virtamo. Dimensioning of data networks: a flow-level perspective. European Transactions on Telecommunications, 20(6):549-563, 2009.

[22] J. Mo and J. Walrand. Fair end-to-end window-based congestion control. IEEE/ACM Transactions on Networking, 8(5):556-567, Oct. 2000. 